\title{
Der (elektrische) Planetenmotor - Eine unkonventionelle Kombination von Elektromotor und Planetengetriebe
}

\author{
M. Schrödl OVE, IEEE
}

In der Arbeit wird der (elektrische) Planentenmotor, ein neues Konzept einer integrierten Multi-Elektromaschine mit einem Planetengetriebe, vorgestellt. Für kompakte Antriebe wird häufig eine hochdrehende elektrische Maschine mit einem nachgeschalteten Getriebe zur Reduktion der Drehzahl eingesetzt. Dabei stellen Motor und Getriebe getrennte Funktionseinheiten dar, die getrennt entworfen und betrieben werden. Die neue Motorstruktur spaltet den Rotor in mehrere Teilrotoren auf, die mechanisch verkoppelt sind und sich ein gemeinsames Wicklungssystem teilen. Nach der Herleitung der Struktur werden das Regelkonzept präsentiert und die Eigenschaften des Antriebs anhand eines Prototypen verifiziert. Die Multirotor-Anordnung verhält sich wie eine einzelne Drehstrommaschine und kann mit einem konventionellen Umrichter betrieben werden. Die Erweiterungsmöglichkeiten des Konzepts auf verschiedene modifizierte Strukturen wird vorgestellt.

Schlüsselwörter: Planetenmotor; Multi-Rotor-Maschine; Planetengetriebe; sensorlose Regelung

The (electrical) planetary motor-an unconventional combination of electric motor and planetary gear box.

In this contribution, a novel multi-machine structure, called "Planetary Motor", is presented. It combines a multi-rotor electric motor with a planetary gear function. Compact drives are frequently built-up using a high-speed electrical motor, combined with a gear box for reducing angular speed. Motor and gear box are separate functional units, which can be constructed and operated seperately. The novel motor structure splits up the rotor in a multi-rotor arrangement which is mechanically coupled and interacting with a common winding system. After describing the motor structure, a control scheme is presented. The multi-rotor arrangement behaves like a single a.c. motor and can be supplied by a conventional inverter. The properties of the drive are explained and verified by a prototype. Further possibilities of modifying the structure are given.

Keywords: Planetary motor; multi-rotor machine; planetary gear; sensorless control

\section{Einleitung und Problemstellung}

Kompakte elektrische Antriebe werden häufig mit hochdrehenden elektrischen Maschinen kombiniert mit einem untersetzenden Getriebe ausgeführt, um hohes Drehmoment bei geringer Baugröße zu erzielen. Die klassische Lösung ist, die Abtriebswelle der elektrischen Maschine als Eingang in ein Stirnrad- oder Planetengetriebe zu verwenden. Motor und Getriebe stellen daher getrennte Funktionseinheiten dar.

Der vorliegende Aufsatz zeigt einen neuen Ansatz zum kombinierten Aufbau einer Motor/Getriebe-Einheit, wobei der Motor als ein verteiltes System mit mehreren Rotoren und das Getriebe als Planetengetriebe ausgeführt sind [1]

In der folgenden Herleitung der Struktur wird die Idee Schritt für Schritt beschrieben.

\section{Herleitung der neuen Maschinenstruktur}

Die Überlegung startet anhand einer einfachen zweipoligen elektrischen Maschine mit einer dreisträngigen Wicklung, bestehend aus je einem Zahn pro Strang. (Bruchlochwicklung mit Lochzahl $q=1 / 2$ ). Ohne Beschränkung der Allgemeinheit sei ein permanentmagneterregter Rotor angenommen. Die Stränge sind geometrisch gemäß Abb. 1 angeordnet.

Nun werden vier gleichartige spiegelsymmetrische Motoren entsprechend Abb. 2 angeordnet. Die Magnetisierungsrichtung der Ro-

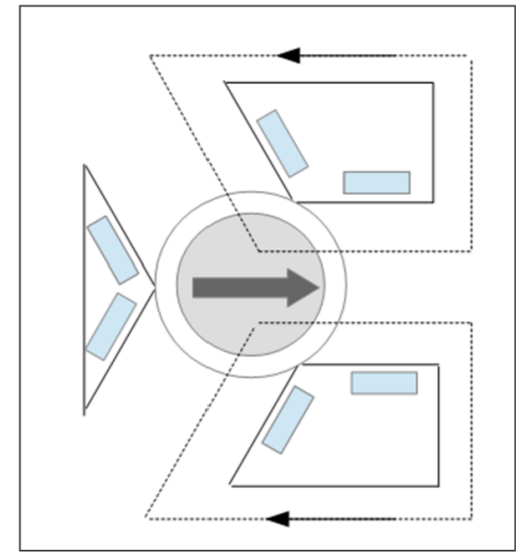

Abb. 1. Ausgangspunkt: Eine dreisträngige zweipolige Drehstrommaschine (z. B. mit Permanentmagneterregung im Rotor, magnetische Feldlinien mit Flussdichte-Richtungspfeilen symbolisch angedeutet)

Schrödl, Manfred, Institut für Energiesysteme und Elektrische Antriebe, Technische Universität Wien, Gußhausstraße 25-29/370, 1040 Wien, Österreich

(E-Mail: Manfred.schroed|@tuwien.ac.at) 

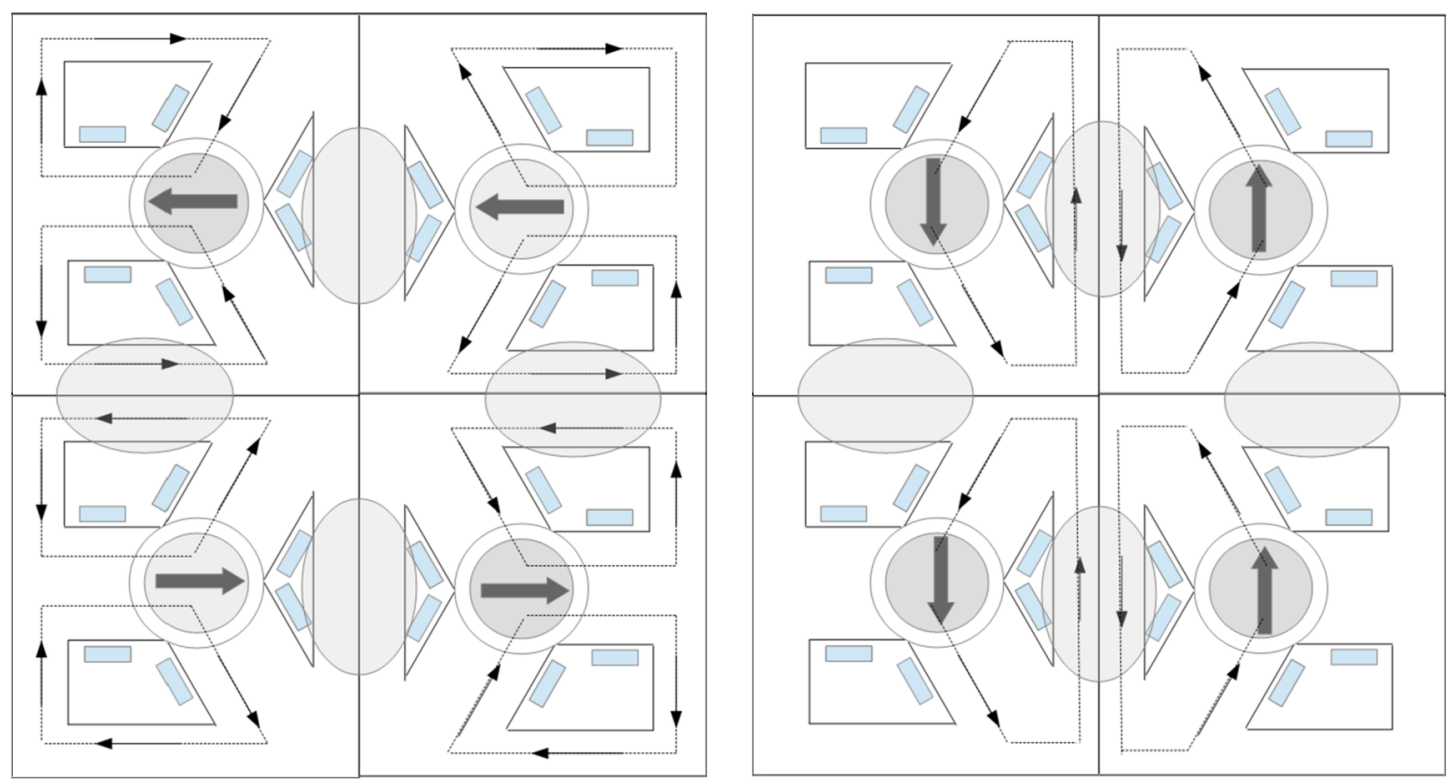

Abb. 2. Symmetrische Anordnung von vier Maschinen (Graue Bereiche: Magnetfeld wird kompensiert bzw. ist nicht vorhanden)
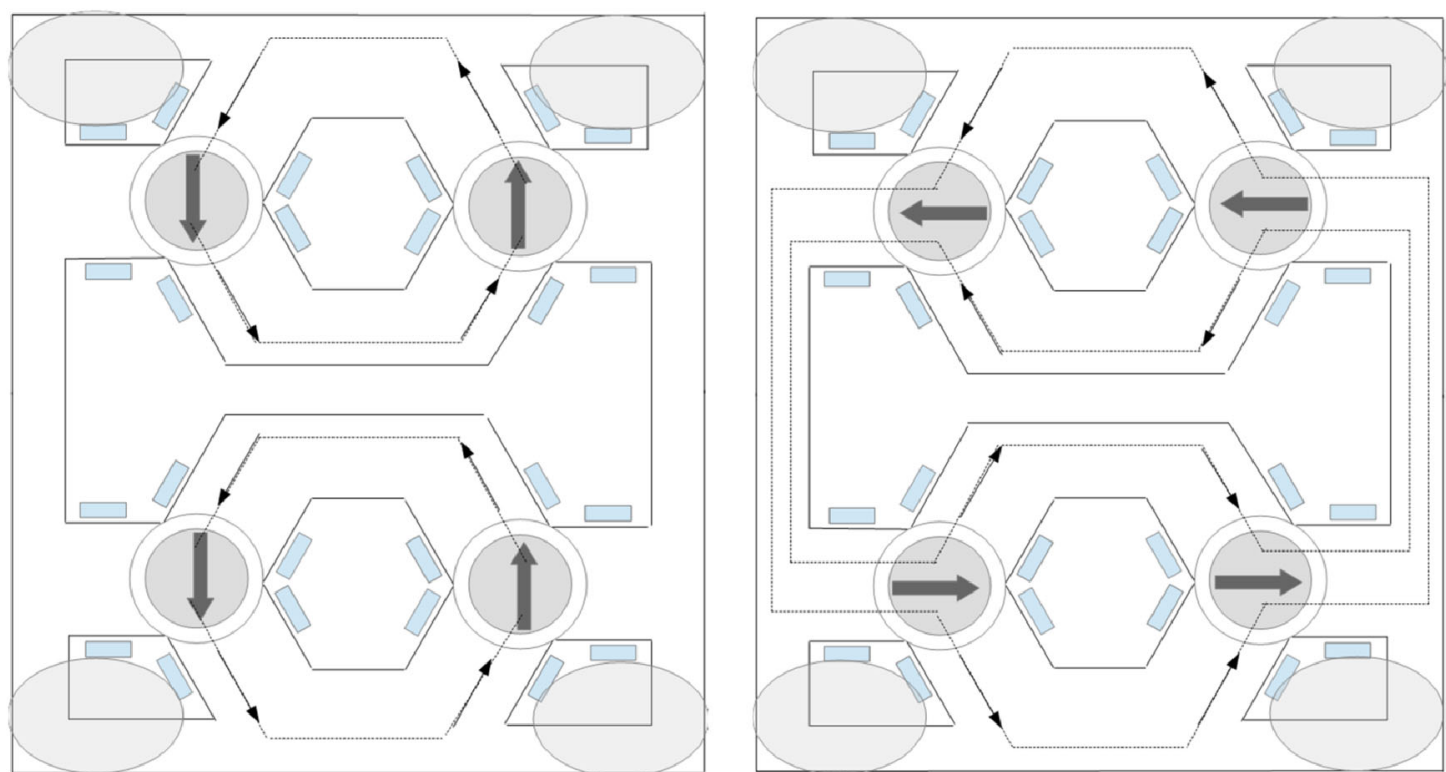

Abb. 3. Reduktion der Aktivmasse durch Elimination von magnetisch inaktiven Abschnitten (graue Bereiche). (a) Vertikale und (b) horizontale Rotorpositionen

toren sei waagrecht angenommen, wobei die Magnetisierungsrichtung der oberen und unteren Motoren gegenläufig ist:

(a) Horizontale Rotorpositionen

(b) Vertikale Rotorpositionen, erzielt durch geeignete $\pm 90 \mathrm{Grad}$ Drehungen der Rotoren gegenüber a)

Wenn die Motoren aneinandergefügt werden, sind einige Abschnitte magnetisch nicht nötig. (Abb. 2a). Nun werden die Rotoren um $\pm 90^{\circ}$ gedreht, wobei benachbarte Rotoren gegenläufig verdreht werden (Abb. 2b). Die Rotoren erzeugen nun eine magnetische Feldverteilung, in der wiederum die gleichen Abschnitte magnetisch nicht nötig sind. Daher kann die Gesamtstruktur vereinfacht werden, wodurch sich die Aktivmasse und die Eisenverluste im Vergleich zur Ausgangssituation mit vier getrennten Maschinen reduzieren. Da die Eckbereiche der Anordnung ebenfalls magnetisch unbelastet sind (Abb. 3a, b), kann eine weitere Vereinfachung von Komplexität und Masse erreicht werden (Abb. 4).

In Abb. 3 sind die resultierenden symbolischen magnetischen Feldlinien für vertikale (a) und horizontale (b) Rotorpositionen dargestellt. Es zeigt sich, dass die Eckgebiete magnetisch nicht benötigt werden. Durch die Elimination der Eckgebiete (siehe graue Bereiche in Abb. 3) können benachbarte Spulen (die vom gleichen magnetischen Fluss durchsetzt werden) entlang der Eisenabschnitte zusammengeschoben werden. Dadurch kann die Anzahl der Spulen von 12 auf 6 reduziert werden. Die vier Teilmotoren teilen sich somit insgesamt 6 Spulen, wobei jeweils 2 einem Strang zugeordnet sind. 

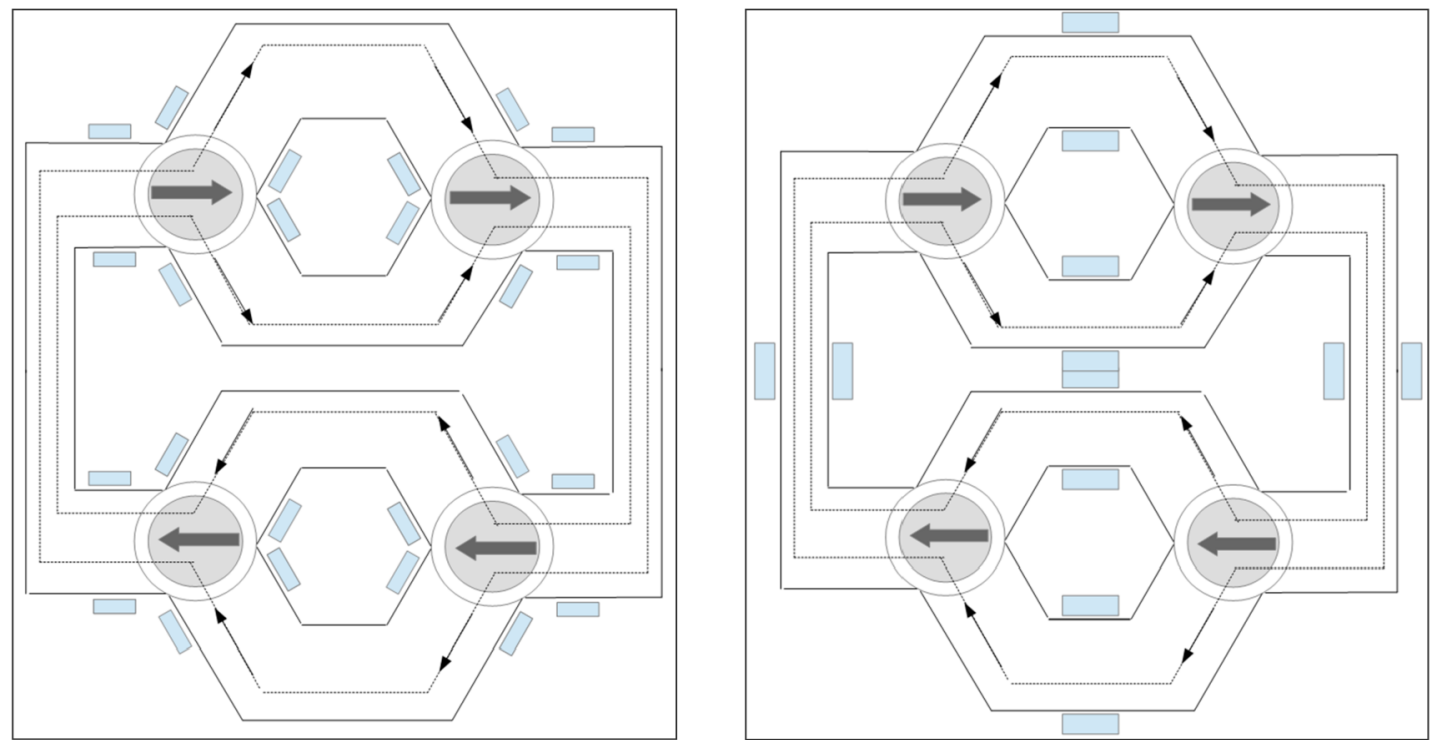

Abb. 4. (a) Elimination der Eckbereiche (graue Bereiche in Abb. 3). (b) Zusammenschieben von benachbarten Spulen und Reduktion der Spulenzahl von 12 auf 6
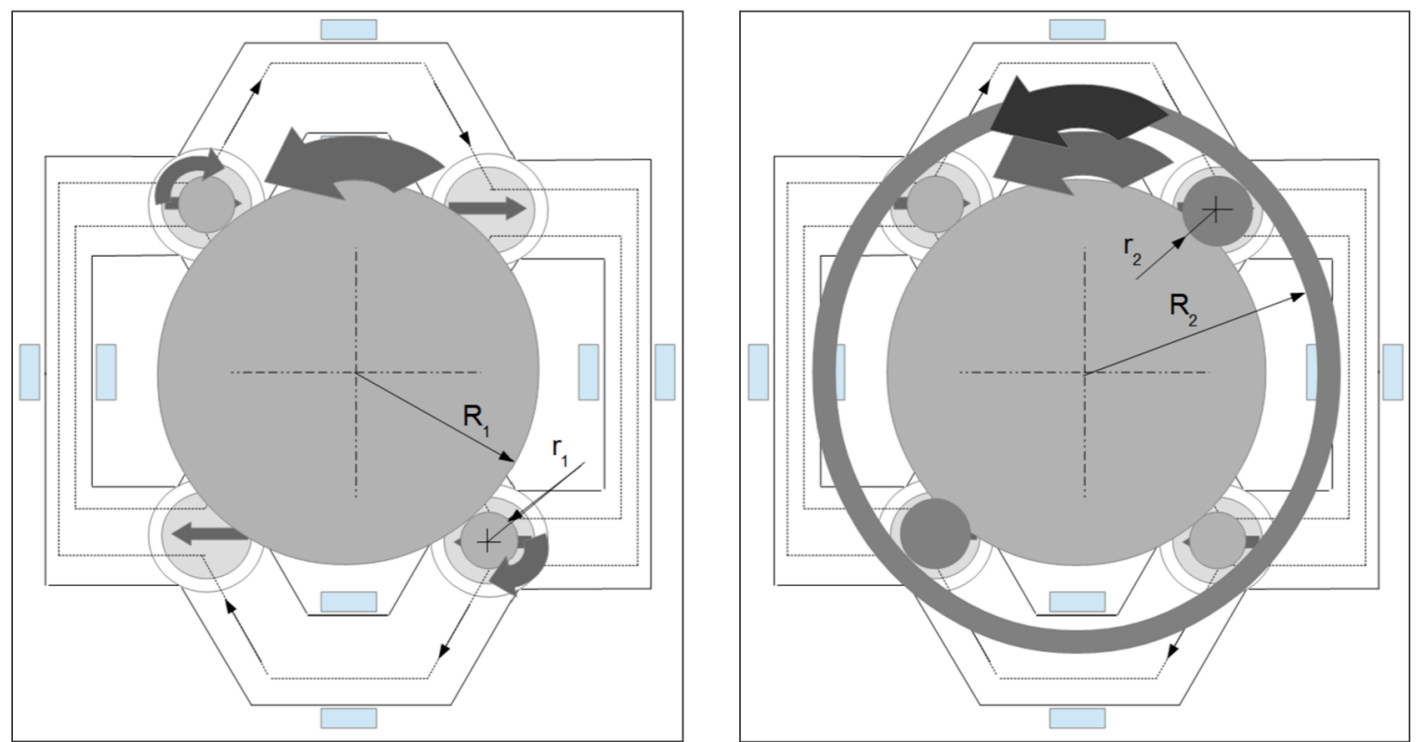

Abb. 5. Kopplung diagonaler Planetenrotoren über ein Sonnenrad mit Außenverzahnung (a) bzw. Innenverzahnung (b)

Durch die obigen Reduktionsschritte in der Geometrie entsteht eine Vier-Rotor-Maschine mit nur sechs konzentrierten Spulen. Im nächsten Schritt werden die Rotoren mechanisch verkoppelt, wobei benachbarte Rotoren in die Gegenrichtung rotieren müssen. Diese mechanische Kopplung wird in der Art eines Planetengetriebes realisiert, wobei jeweils zwei gegenüberliegende "Planetenrotoren" über Zahnräder in ein zentrales Sonnenrad unter Realisierung einer Getriebeübersetzung eingreifen (Abb. 5). Dabei ist das Übersetzungsverhältnis in weiten Grenzen zwischen Über- und Untersetzungen wählbar. Typischerweise wird bei elektrischen Antrieben eine Untersetzung angestrebt, weil häufig hohes Drehmoment bei relativ niedriger Drehzahl am Abtrieb gefordert wird (Abb. 5a). Antriebe mit einer Übersetzung zu höheren Abtriebsdrehzahlen des Sonnenrades sind seltener, aber auch darstellbar. Die umgekehrte Drehrich- tung der verbleibenden Planeten kann durch eine Innenverzahnung des Sonnenrades realisiert werden (Abb. 5b).

Unter Verwendung einer Übersetzung $i$, mit

$$
i=\frac{R_{1}}{r_{1}}=\frac{R_{2}}{r_{2}}
$$

rotieren beide Teile (mit Außen- bzw. Innenverzahnung) des Sonnenrades mit der gleichen Winkelgeschwindigkeit. Die Planeten rotieren mit betragsmäßig gleicher Winkelgeschwindigkeit, aber unterschiedlichen Drehrichtungen von benachbarten Rotoren. Für eine kollisionsfreie Realisierung der Zahnräder müssen die Planeten mit unterschiedlichen Drehrichtungen geometrisch entkoppelt werden. Dies kann durch axiale Versetzung der beiden Planetengruppen (und der zugehörigen Sonnenradteile) oder auch durch radiale Entkopplung (gleichzeitige Erhöhung der Radien $R_{2}$ und $r_{2}$ um den gleichen 

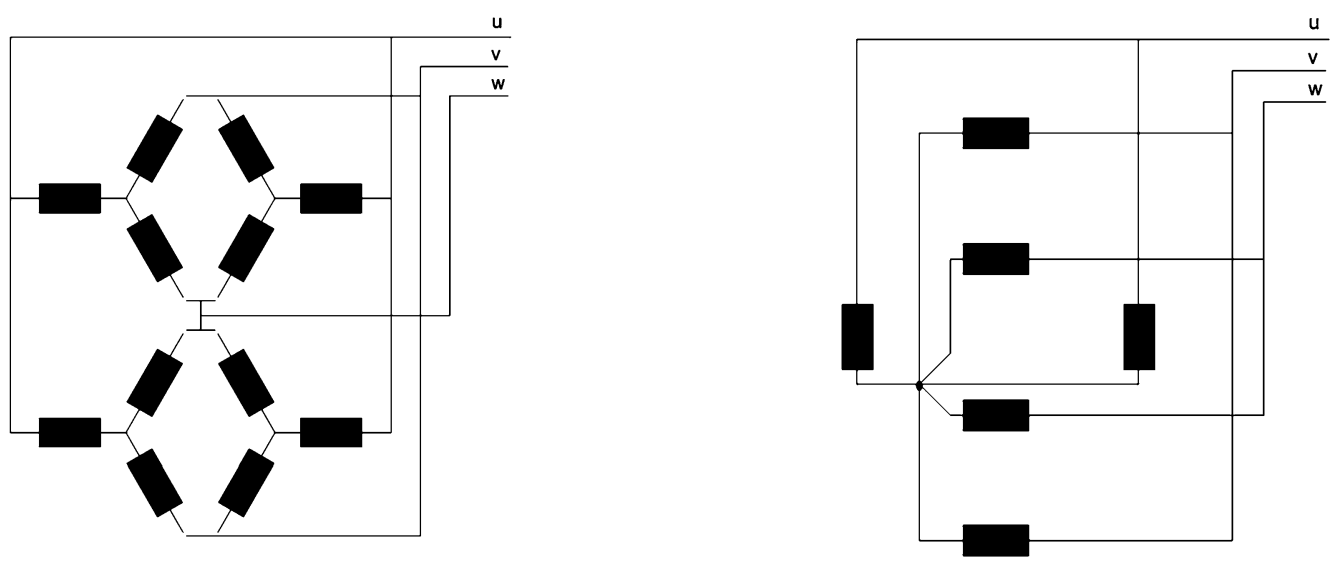

Abb. 6. (a) Originale Vierfach-Sternschaltung der Teilmotoren. (b) Planetenmotor mit Sternschaltung der Stränge, Strang-Teilspulen parallel (Alternativ: Serienschaltung möglich)

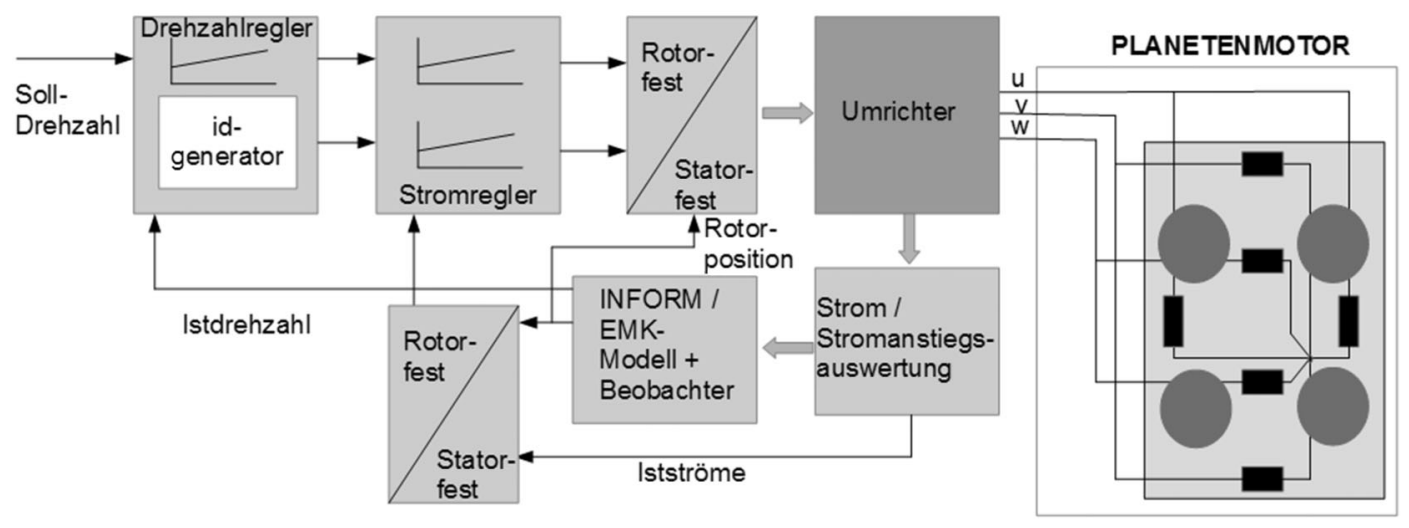

Abb. 7. Sensorlose feldorientierte INFORM/EMK-Regelung des Planetenmotors

Faktor) erzielt werden. In letzterem Fall liegen die Achsen der vier Rotoren nicht mehr in den Ecken eines Quadrats, sondern in den Ecken eines Rhombus oder Rhomboids. Diese Variante wurde aufgebaut und getestet (Abb. 8).

\section{Elektrische Verschaltung des Planetenmotors}

Ausgehend von der Viermotor-Topologie mit 12 Spulen seien die Teilmotoren beispielsweise in Stern geschaltet. (Abb. 6a). Die vereinfachte Spulenanordnung des Planetenmotors kann z. B. in Stern, Dreieck oder offener $\mathrm{H}$-Brücke geschaltet werden. Weiters können die zwei Teilspulen der Stränge in Serie oder parallel geschaltet werden. In Abb. 6b ist ein Schaltungsbeispiel mit zwei parallelen Spulen pro Strang, sowie eine Sternschaltung der drei Stränge $u, v, w$ gezeigt.

\section{Regelung des Planetenmotors}

Wie in Abb. 6 gezeigt, bildet der Planetenmotor ein klassisches dreisträngiges System. Obwohl die Geometrie der elektrischen Aktivteile nicht zylindrisch ist, verhält sich die Maschine wie ein symmetrischer dreisträngiger Motor. Daher kann ein üblicher dreisträngiger Umrichter mit konventioneller Regelung (z. B. feldorientiert) verwendet werden. (Abb. 7).

Wie in Abb. 7 dargestellt, kann eine besonders wirtschaftliche Realisierung des Planetenmotors unter Verwendung von sensorlosen Regelverfahren dargestellt werden [2]. Sensorlose Verfahren (kein mechanischer Geber nötig) sind seit vielen Jahren Gegenstand von Forschung und bereits in diversen Anwendungen im Einsatz [3-5].

\section{Vorteile des Planetenmotors}

\subsection{Reduzierte Masse der Aktivteile}

Wie gezeigt wurde, ermöglicht die Konfiguration eine erhebliche Reduktion der magnetisch aktiven Teile im Vergleich zu den einzelnen vier Motoren.

\subsection{Reduzierte Anzahl von Spulen und einfache Fertigung}

Die ursprünglichen 12 Spulen wurden zu sechs einfachen Spulen mit rechteckigem Querschnitt vereinfacht. Die Spulenenden können in der gleichen Ebene ausgeführt werden. Dies ermöglicht eine einfache (vollautomatische) Montage von Motor und Elektronikteil (Abb. 8b). Die zwei Spulen je eines Stranges können in Serie oder parallel geschaltet werden. Weiters können die Stränge in Dreieck oder Stern geschaltet werden.

\subsection{Deutliche Erhöhung der installierbaren Leistung bei Hochdrehzahlrotoren}

Betrachten wir einen Kompaktantrieb bestehend aus Hochdrehzahlmotor und Untersetzungsgetriebe, etwa einen Elektroauto-Antrieb (Abb. 9)

Bei gegebenem Rotordurchmesser wird die Umfangsgeschwindigkeit aufgrund der Festigkeitseigenschaften des Rotormaterials limi- 

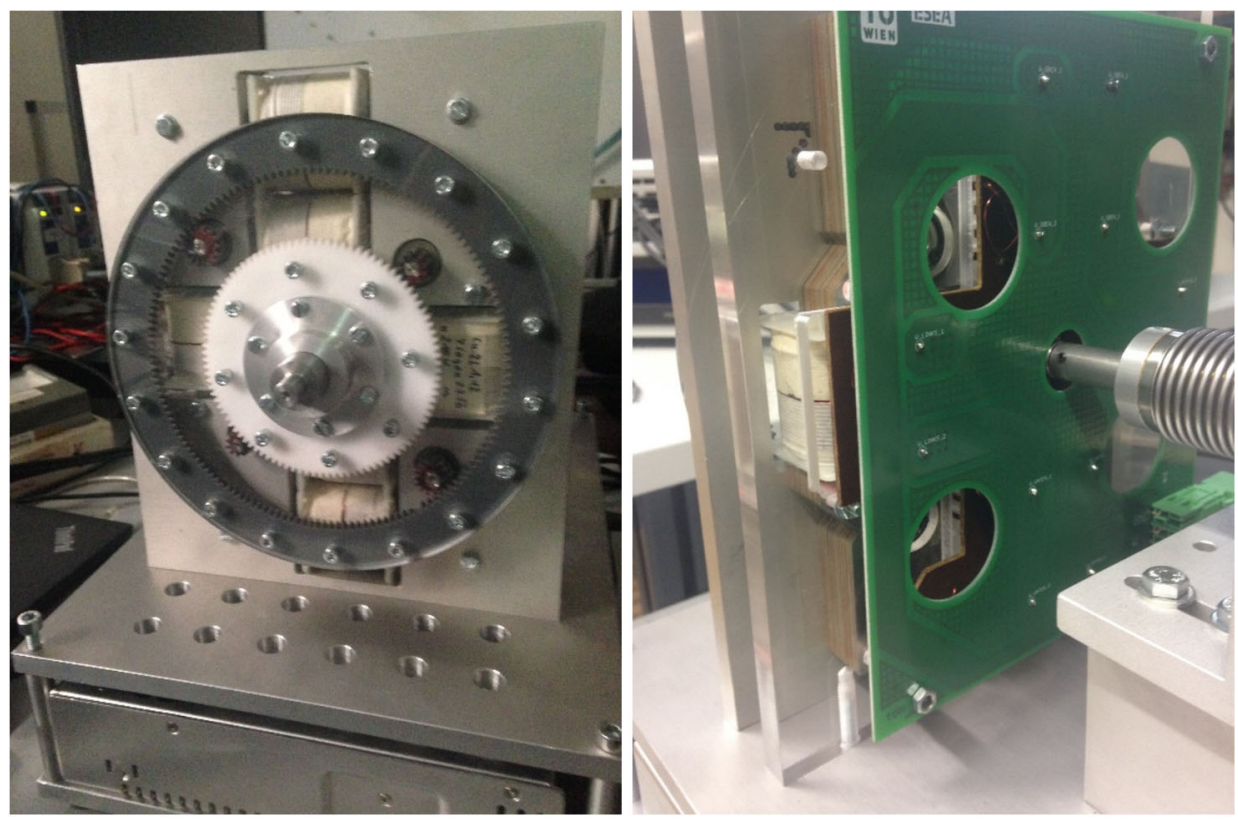

Abb. 8. (a) Planetenmotor mit rhombusförmiger Anordnung der Planetenachsen und Untersetzung $i=10$. (b) Einfache Montage der Elektronik und Kontaktierung der Spulen an der Rückseite des Planetenmotors
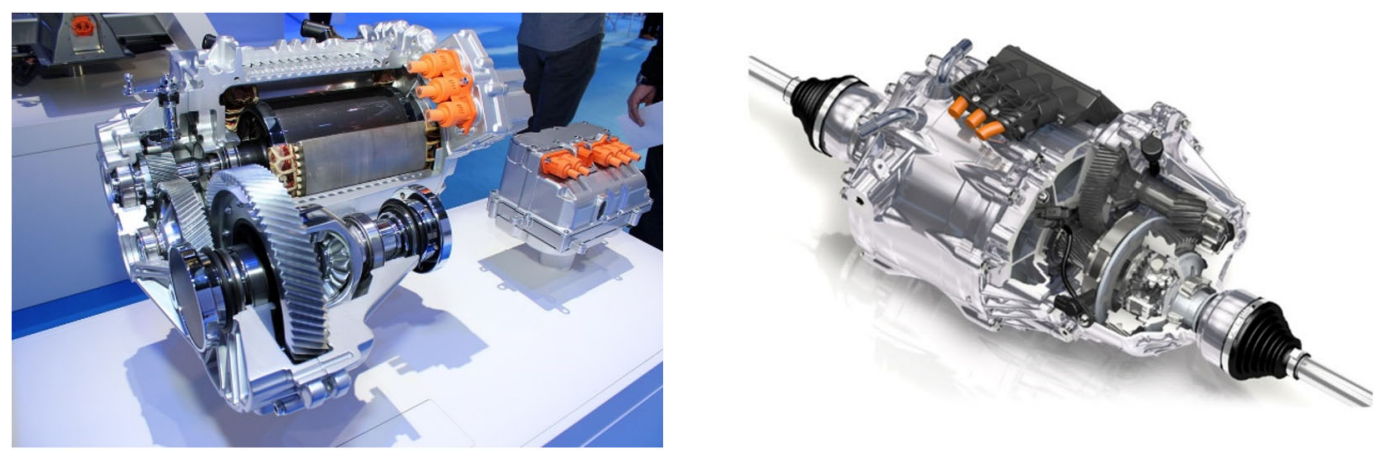

Abb. 9. (a) Klassische Antriebseinheit für ein Elektrofahrzeug mit Elektromotor, Stirnradgetriebe und Differenzialgetriebe (Foto: VW). (b) Elektrische Antriebseinheit mit koaxialem Abgang der Halbachsen aus dem Differenzialgetriebe durch die hohle Motorwelle (Zeichnung: GKN)

tiert. Typischerweise können Umfangsgeschwindigkeiten im Bereich von $100-200 \mathrm{~m} / \mathrm{s}$ wirtschaftlich dargestellt werden [6]. Unterstellt man die gleiche spezifische Schubkraft im Luftspalt sowohl des ursprünglichen klassischen Motors als auch des Planetenmotors (gleiche Flussdichte-Grundwelle und gleicher Strombelag), so können wir den Originalrotor (Durchmesser $D$ ) durch vier in Summe flächengleiche Planetenrotoren (Durchmesser jeweils D/2) ersetzen. Jeder Planetenrotor liefert dann $\frac{1}{4}$ des ursprünglichen Drehmomentes (halbe Umfangskraft wegen halber Umfangsfläche im Luftspalt; halber Radius). In Summe liefern die vier Planetenrotoren das gleiche Drehmoment wie der Originalrotor. Bei gleicher Leistung von Originalmotor und Planetenmotor bedeutet dies gleiche Drehzahl des ursprünglichen Rotors und der Planetenrotoren. Aufgrund des halben Durchmessers tritt also nur die halbe Umfangsgeschwindigkeit gegenüber dem ursprünglichen Rotor auf. Wird die Leistungsgrenze durch die Umfangsgeschwindigkeit festgelegt, kann diese im Planetenmotor um den Faktor 2 erhöht werden, bis die gleiche Umfangsgeschwindigkeit auftritt.
Vereinfachte Planetengetriebestufe Im Vergleich zu einem normalen Planetengetriebe weist die Planetenstufe des Planetenmotors einige Vorteile auf. Die vier Rotoren erzeugen praktisch das gleiche Drehmoment, wodurch automatisch eine gleichmäßige Krafteinleitung in die Zähne des Sonnenrades gewährleistet ist. Es ist also aus Sicht der Kraftaufteilung auf die Planeten keine besondere Präzision bei der Zahnfertigung nötig. Weiters erfolgt die Kraftumsetzung beim Planetenmotor nur in einem Kontaktpunkt pro Planeten-Zahnrad (im Gegensatz zu zwei Kontaktstellen beim klassischen Planetengetriebe), wodurch der Wirkungsgrad des Getriebes deutlich besser als beim normalen Getriebe ist.

\section{Messungen am Prototypen}

Um die Funktionsweise des Planetenmotors zu verifizieren, wurde ein 1 kW-Prototyp aufgebaut und getestet (Abb. 10a). Das Sonnenrad wurde aus transparentem Material aufgebaut, um eine gute optische Einsicht in das Funktionsprinzip zu bekommen. Der Magnetkreis wurde auf Basis numerischer Simulation ausgelegt. Entsprechend der kleinen Zähnezahl zeigt der Verlauf der induzierten Spannung einen gewissen Oberschwingungsgehalt (Abb. 10b). Die- 

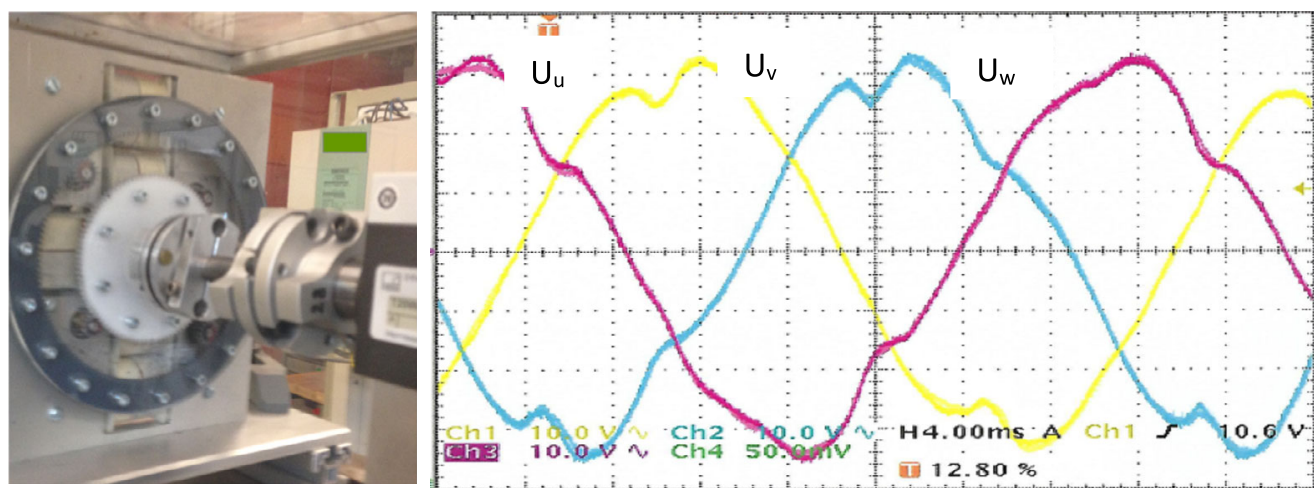

Abb. 10. (a) $1 \mathrm{~kW}$-Planetenmotor am Prüfstand. (b) Verlauf der Strangspannungen $U_{u}, U_{v}, U_{w}$ im Leerlauf bei $2000 \min ^{-1}$ am Planetenrotor (200 $\mathrm{min}^{-1}$ am Abtrieb) ( $y$-Achse: Spannungen $u, v, w$ mit $10 \mathrm{~V} / \mathrm{div}, x$-Achse: Zeit $t$ mit $4 \mathrm{~ms} / \mathrm{div}$ )

ser kann durch Optimierung der Geometrie weiter verringert werden. Anmerkung: Messungen unter Belastung werden in späteren Veröfffentlichungen publiziert.

\section{Vorteilhafte Anwendungsgebiete}

Die Struktur des Planetenmotors eröffnet viele vorteilhafte Anwendungsgebiete. Speziell für Antriebsaufgaben mit einem Untersetzungsgetriebe können sehr kompakte Lösungen mit hohem Automatisierungsgrad der Fertigung dargestellt werden.

\subsection{Windkraftantriebe}

Aus dem Bereich großer Leistungen seien Windkraftantriebe erwähnt, wo die Hauptwelle mit den Rotorblättern an das Sonnenrad eines Planetenmotor/-generators angefügt werden kann und die elektrischen Generatoren der Planetenmaschine mit entsprechend erhöhter Drehzahl laufen, wodurch die Baugröße der Generatoren und das häufig eingesetzte Getriebe deutlich günstiger realisiert werden können.

\subsection{Außenläuferanwendungen}

Bei Außenläufer-Antrieben, wie z. B. Trommelmotoren, Radnabenmotoren, Rohrmotoren etc. kann das Sonnenrad des Planetenmotors direkt mit dem rotierenden Außenteil verbunden werden, wodurch sehr kompakte Lösungen möglich sind.

\subsection{Elektrofahrzeug-Antriebe}

Elektrofahrzeuge haben Antriebseinheiten bestehend aus klassischem Elektromotor und Getriebestufe. In Abb. 9a) ist die Antriebseinheit eines VW E-Golf gezeigt. Die elektrische Maschine ist über ein zweistufiges Stirnradgetriebe und ein Differenzialgetriebe an die beiden Halbachsen gekoppelt. Alternativ kann der Antrieb koaxial aufgebaut werden (z. B. Lösung von GKN, Abb. 10b), wo die Motorwelle als Hohlwelle ausgeführt ist und die elektrische Maschine mit einem Planetensatz und einem folgenden Differenzialgetriebe verbunden ist. Das Differenzialgetriebe treibt die beiden Halbachsen an, wobei eine Halbachse durch die Hohlwelle des Motors geführt ist (Transaxle-Lösung).

Führt man diese Grundstruktur als Planetenmotor aus, erhält man eine sehr kompakte Einheit (Abb. 11). Das Sonnenrad des Planetenmotors ist mit einer Hohlwelle ausgeführt und direkt mit dem Differenzialgetriebe konstruktiv verbunden. Damit können diverse mechanische Komponenten eingespart werden.

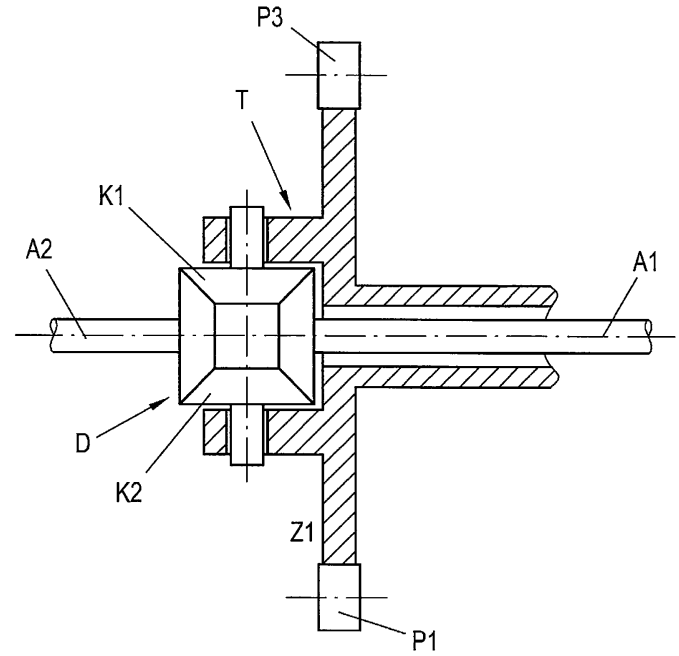

Abb. 11. Planetenmotor mit Differenzialgetriebe $D$ (Kegelradträger $T$, Kegelräder $K 1, K 2$ ), funktionell kombiniert mit dem Sonnenrad $Z 1$. Planeten $P 1, P 3$ (ohne angeschlossene Rotoren des elektrischen Teils dargestellt). Halbachsen $\boldsymbol{A 1}, \boldsymbol{A} \mathbf{2}$, die mit den Antriebsrädern verbunden werden

\section{Ausblick: Planetenmotor-Strukturen mit anderen}

\section{Polpaarzahlen und Planetenzahlen}

Die hergeleitete Grundstruktur kann auch an andere Polpaar- und Planetenzahlen angepasst werden. Damit können beispielsweise vierpolige Rotoren oder 6 Planeten realisiert werden. Abbildung 12 zeigt die grundsätzliche Motorstruktur für eine Anordnung mit vier vierpoligen Rotoren (Spulen nicht dargestellt). Die Rotoren drehen wieder mit entgegengesetzter Drehrichtung und gleichem Drehzahlbetrag. Die Pfeile in Abb. 12 deuten den magnetischen Flussverlauf bei der gewählten Rotorstellung an.

Interpretiert man die Struktur gemäß Abb. $4 b$ als Serienschaltung von 2 gleichartigen Strukturmodulen, so kann man aus diesem Strukturmodul verschiedenste Planetenmotoren konstruieren. Als Beispiel ist in Abb. 13 ein Planetenmotor mit 6 Planeten realisiert, indem 3 Strukturmodule zusammengefügt wurden. Benachbarte Planeten rotieren wiederum in Gegenrichtung.

\section{Zusammenfassung}

In der Arbeit wurde der (elektrische) Planentenmotor - ein neues Konzept einer integrierten Multi-Elektromaschine mit einem Plane- 


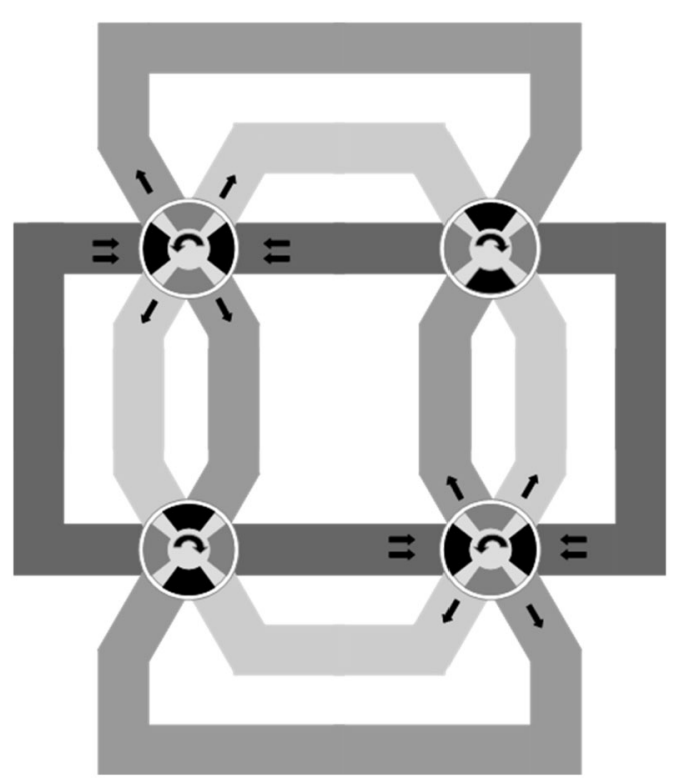

Abb. 12. Planetenmotor mit vierpoligen Rotoren (Stränge $u, v, w$ in unterschiedlichen Graustufen, Pfeile deuten magnetische Flussdichte mit Richtung an)

tengetriebe - vorgestellt. Nach der Herleitung der Struktur wurde ein Prototyp aufgebaut, das Regelkonzept präsentiert und die Eigenschaften des Antriebs verifiziert. Die ausgeführte hochdynamische feldorientierte Regelung wurde sensorlos auf Basis des INFORM ${ }^{\circledR}$ Verfahrens in Kombination mit einem EMK-Modell realisiert und im gesamten Betriebsbereich einschließlich Stillstand und kleinen Drehzahlen getestet. Die Erweiterungsmöglichkeiten des Konzepts auf verschiedene modifizierte Strukturen wurde vorgestellt. Am Institut werden weitere Varianten des Planetenmotors aufgebaut und in nachfolgenden Veröffentlichungen präsentiert werden.

\section{Danksagung}

Open access funding provided by TU Wien (TUW).

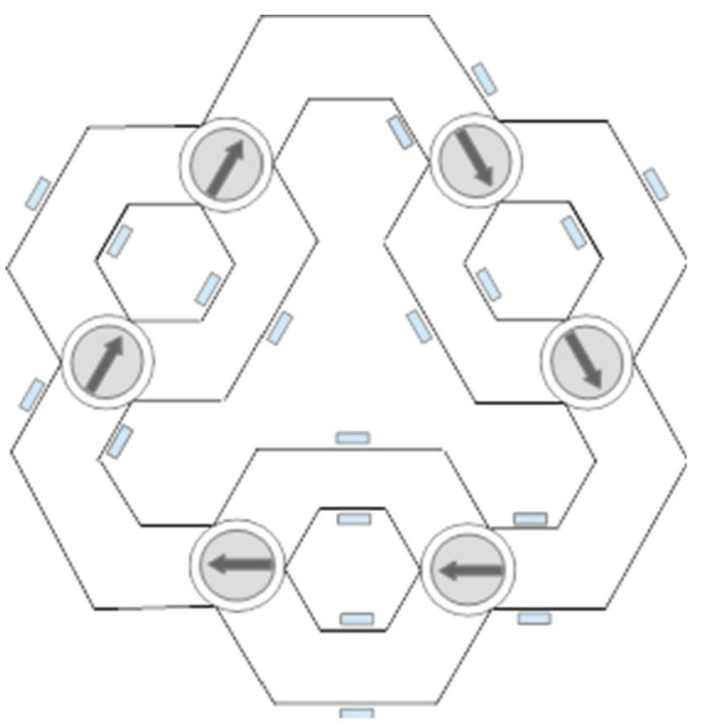

Abb. 13. Struktur mit 6 zweipoligen Planeten

Open Access This article is distributed under the terms of the Creative Commons Attribution 4.0 International License (http://creativecommons.org/ licenses/by/4.0/), which permits unrestricted use, distribution, and reproduction in any medium, provided you give appropriate credit to the original author(s) and the source, provide a link to the Creative Commons license, and indicate if changes were made.

\section{Literatur}

1. Schrödl, M. Elektrisches Maschinensystem. Österreichische Patentanmeldung Nr. A50594/2016.

2. Rajashekara, K. et al. (Hrsg.) (1996): Sensorless control of AC motor drives. New York: IEEE Press, PC3996, ISBN0-7803-1046-2.

3. Schrödl, M. (1992): Sensorless control of AC machines. Habilitationsschrift, VDIFortschrittsberichte Nr. 117, Reihe (21).

4. Preusser, T. (2002): Neues sensorloses Regelverfahren für Synchronmaschinen. Antriebstechnik, 41, 21-23. $\mathrm{Nr} 7$.

5. Consoli, A., Scarcella, G., Testa, A. (2001): Industry applications of zero speed sensorless control techniques for PM synchronous motors. IEEE Trans. Ind. Appl., 37(2), $513-521$.

6. Viggiano, F. (1992): Aktive Magnetische Lagerung und Rotorkonstruktion Elektrischer Hochgeschwindigkeits-Antriebe. Dissertation, ETH Zürich. ADAG AG, Zürich.

\section{Autor}

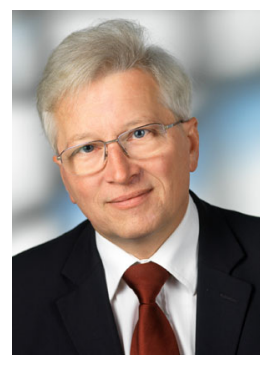

\section{Manfred Schrödl}

Studium der Elektrotechnik an der Technischen Universität Wien, Österreich, Studienzweig Industrielle Elektronik und Regelungstechnik; Abschluss 1982. Universitätsassistent am Institut für Elektrische Maschinen und Antriebe der TU Wien, 1987 Promotion zum Dr. techn., 1992 Habilitation für Elektrische Antriebe und Leistungselektronik in der Antriebstechnik. 1993 bis 1996 Leiter F\&E bei
Elin Verkehrstechnik Wien, 1996 bis 1998 Bereichsleiter Zentrale Technik bei Flender ATB, Spielberg, Steiermark. Seit 1998 Ordentlicher Universitätsprofessor für Elektrische Antriebe und Maschinen sowie Vorstand des Instituts für Energiesysteme und Elektrische Antriebe an der TU Wien. 